\title{
Metabolic Abnormalities in Tick-Borne Encephalitis: A Mini-Review
}

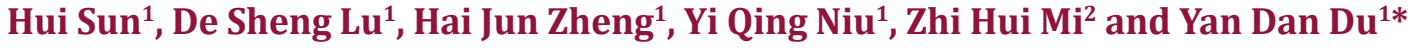 \\ ${ }^{1}$ Department of Clinical Laboratory, Inner Mongolia Forestry General Hospital (The second Clinical Medical School of Inner \\ Mongolia, University for the Nationalities), Hulunbuir, Inner Mongolia, China
}

2Inner Mongolia Di An Feng Xin Medical Technology Co., LTD, Huhhot, Inner Mongolia, China

*Corresponding author: Yan Dan Du, Inner Mongolia Di An Feng Xin Medical Technology Co., LTD, Huhhot, Inner Mongolia, China

\section{ARTICLE INFO}

Received: 蔧 June 01, 2021

Published: 絊 June 10, 2021

Citation: Hui Sun, De Sheng Lu, Hai Jun Zheng, Yi Qing Niu, Zhi Hui Mi, Yan Dan Du. Metabolic Abnormalities in Tick-Borne Encephalitis: A Mini-Review. Biomed J Sci \& Tech Res 36(3)-2021. BJSTR. MS.ID.005848.

\section{ABSTRACT}

Tick-borne encephalitis (TBE) is a debilitating, heterogeneous disease associated with myriad symptoms that vary depending on the infection subtype and duration. There are no known biomarkers for TBE, largely due to a lack of knowledge of its pathogenesis. Numerous studies have sought potential biomarkers for the disease. This mini review concisely outlines the current state of research on metabolic abnormalities in TBE, and may provide a foundation for further studies of the disease. Research progress in key areas such as the immune response and metabolites of dysregulation, is also described. The studies outlined here suggest potential biomarkers for TBE. Future research at multiple research centers should focus on firmly establishing these biomarkers. Whether the metabolic changes noted in this mini review are a cause or a consequence of TBE must also be verified.

Abbreviations: TBEV: Tick-Borne Encephalitis Virus; PLs: Phospholipids; DHA: Docosahexaenoic Acid; AA: Arachidonic Acid

\section{Introduction}

Tick-Borne Encephalitis (TBE) is a debilitating sudden-onset disease that affects the central nervous system. There are currently no definitive biomarkers for the disease and no therapeutic targets. TBE is usually treated with antipyretics and analgesics. This mini review outlines the progress made toward identifying potential metabolic biomarkers of TBE.

\section{Immune Response and Metabolites Dysregulation}

It is thought that Tick-Borne Encephalitis Virus (TBEV) infection activates the immune system. In humans, TBEV infection causes early immunological reactions that are initially pro-inflammatory, and then anti-inflammatory [1]. Numerous immune dysregulation phenotypes have been reported in patients infected with viruses, including TBEV $[2,3]$. After viral invasion, the body makes a series of adjustments to maintain homeostasis and health. Numerous biochemical compounds are released, leading to changes in amino acids, Phospholipids (PLs), sphingomyelins, and triglycerides.
Abnormalities in these metabolites may explain the typical findings of TBEV infection, i.e., fever, nausea, and encephalitis.

Many inflammatory and anti-inflammatory metabolites have been implicated in viral infection, including Arachidonic Acid (AA) and polyunsaturated fatty acids (PUFAs). AA, a potential biomarker for COVID-19, is involved in the passivation of enveloped viruses, such as influenza virus [4]. A relatively small study of 60 TBEV patients and 56 controls found that AA and PUFAs levels differed between the groups. Moreover, significant decreases in AA and Docosahexaenoic Acid (DHA) were observed, in contrast to another study [5]. Two of the most recent studies of AA provided conflicting results: one found decreased levels in herpes virus infection patients [6], while we found an increasing trend during the pyrogenic phase of coronavirus infection [7]. More recent studies have focused on the role of PLs in the pathogenesis of viral diseases, where PLs are potential therapeutic targets [8]. One recent study investigated the connection between PLs and disease severity and showed that lipid 
species differed significantly in comparison to membranes from uninfected cells [9]. PLs dominate cell membranes and may disrupt cell homeostasis, thus causing physiological and pathological changes, and impairing barrier function [10].

Promising studies of immune dysregulation in TBE have implicated amino acids [11]. Amino acids initiate biochemical reactions and changes. Moreover, glutamate and aspartate are primary excitatory neurotransmitters, and may mediate the association between neurotropic viral infection and metabolite dysregulation [12]. There is some evidence that TBEV may affect, at least in part, energy metabolism. Studies of TBE have reported markers of energy metabolism dysfunction, such as abnormal acylcarnitine production and altered lipid metabolism, especially fatty acid $\beta$-oxidation. Changes in serum acylcarnitine levels suggest mitochondrial dysfunction, which is associated with altered fatty acid transport [13]. As well as showing significant differences between TBE patients and healthy controls, abnormal acylcarnitine metabolism was seen in HIV-exposed uninfected newborns [14].

\section{Metabolomics}

Few comprehensive metabolomic studies of TBE have been performed. One study assessed metabolites in enrichment pathways in the serum of 59 patients and 30 healthy controls. The results supported the hypothesis of a disordered metabolic state in TBE. The pathways in which metabolite levels were abnormal varied, with some being associated with the immune response. The authors suggested that a distinct metabolic response to viral infection occurs in TBE patients and concluded that serum metabolomics could be used to identify biomarkers of TBEV disease. However, the results were based on a small number of samples.

\section{Conclusion}

The pathogenesis of TBE remains elusive despite efforts to identify biomarkers of the disease. The studies outlined here identified potential biomarkers of TBE, including LPs, fatty acids, amino acids, and energy metabolites. The conflicting results, i.e., lack of reproducibility, make identifying biomarkers difficult. To clarify the mechanisms of the disease, further research into the pathology of TBE, and the role of TBEV, must be conducted at multiple centers to build create foundation for further research [2].

\section{Funding}

This study was funded by grants from and Natural Science Foundation of Inner Mongolia (2019MS08174).

\section{Competing Interests}

The Authors declare that there are no competing interests associated with the manuscript.

\section{References}

1. Mathew A, Rothman AL (2008) Understanding the contribution of cellular immunity to dengue disease pathogenesis. Immunol Rev 225 : 300-313.

2. Du Y, Mi Z, Xie Y, Lu D, Zheng H, et al. (2021) Insights into the molecular basis of tick-borne encephalitis from multiplatform metabolomics. PLoS Negl Trop Dis 15: e0009172.

3. Mohamed Khosroshahi L, Rezaei N (2021) Dysregulation of the immune response in coronavirus disease 2019. Cell Biol Int 45: 702-707.

4. Das UN (2020) Can Bioactive Lipids Inactivate Coronavirus (COVID-19)? Arch Med Res 51(3): 282-286.

5. Luczaj W, Moniuszko A, Jarocka-Karpowicz I, Pancewicz S, Andrisic (2016) Tick-borne encephalitis--lipid peroxidation and its consequences. Scand J Clin Lab Invest 76(1): 1-9.

6. Dl B, Jj S, He K, Ng B (1986) Arachidonic acid metabolism to eicosanoids in herpes virus-infected rabbit cornea. Invest Ophthalmol Vis Sci 27(10): 1443-1446.

7. Yan B, Chu H, Yang D, Sze KH, Lai PM, et al. (2019) Characterization of the Lipidomic Profile of Human Coronavirus-Infected Cells: Implications for Lipid Metabolism Remodeling upon Coronavirus Replication. Viruses 11.

8. Tsai HC, Han MH (2016) Sphingosine-1-Phosphate (S1P) and S1P Signaling Pathway: Therapeutic Targets in Autoimmunity and Inflammation. Drugs 76(11): 1067-1079.

9. Perera R, Riley C, Isaac G, Hopf-Jannasch AS, Moore RJ, et al. (2012) Dengue virus infection perturbs lipid homeostasis in infected mosquito cells. PLoS Pathog 8: e1002584.

10. Volinsky R, Kinnunen PK (2013) Oxidized phosphatidylcholines in membrane-level cellular signaling: from biophysics to physiology and molecular pathology. FEBS J 280: 2806-2816.

11. Huang R, Gao H, Zhang L, Jia J, Liu X, et al. (2012) Borna disease virus infection perturbs energy metabolites and amino acids in cultured human oligodendroglia cells. PLoS One 7: e44665.

12. Zhang CX, Ofiyai H, He M, Bu X, Wen Y, et al. (2005) Neuronal activity regulates viral replication of herpes simplex virus type 1 in the nervous system. J Neurovirol 11(3): 256-264.

13. Breit M, Weinberger KM (2016) Metabolic biomarkers for chronic kidney disease. Arch Biochem Biophys 589: 62-80.

14. Kirmse B, Yao TJ, Hofherr S, Kacanek D, Williams PL, et al. (2016) Acylcarnitine Profiles in HIV-Exposed, Uninfected Neonates in the United States. AIDS Res Hum Retroviruses 32(4): 339-348. 
ISSN: 2574-1241

DOI: 10.26717/BJSTR.2021.36.005848

YanDan Du. Biomed J Sci \& Tech Res

(c) (P) This work is licensed under Creative BY Commons Attribution 4.0 License

Submission Link: https://biomedres.us/submit-manuscript.php

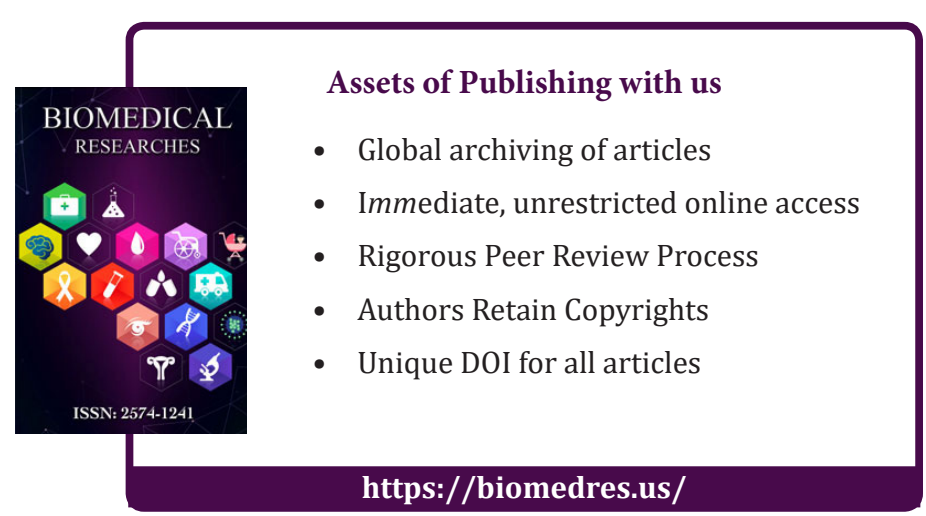

